\title{
New Developments in Therapeutic HPV Vaccines
}

\author{
Jiaying Lin • Jinfeng Xu • Andreas E. Albers • \\ Andreas M. Kaufmann
}

Published online: 26 May 2012

(C) Springer Science+Business Media, LLC 2012

\begin{abstract}
Human papillomavirus (HPV) infection is the central causal factor in cervical cancer. For years efforts were undertaken to control HPV-associated disease by the development of HPV vaccines. While prophylactic HPV vaccination represents a major public health breakthrough, it is limited to new infection by certain HPV types and does not have therapeutic effects or prevent progression of preexisting HPV infections to malignancy. Thus, there is an urgent need for therapeutic HPV vaccines. Therapeutic vaccines aim to induce cytotoxic $\mathrm{T}$ cells, facilitating clearance of HPV high-grade lesions and cancers. HPV E6 and E7 oncogenes represent ideal antigens for therapeutic HPV vaccination because of their constitutive expression in HPV-containing carcinomas and their critical role in the induction and maintenance of HPV-associated disease. This review discusses the most recent advances in HPV therapeutic vaccines and summarizes effects of new basic immunology findings and vaccine delivery technologies.
\end{abstract}

Keywords Cervical cancer - Cervical intraepithelial neoplasia $\cdot \mathrm{CIN} \cdot$ Immunization $\cdot$ Clinical trials · Immunotherapy $\cdot$ Lesions

J. Lin · J. Xu • A. M. Kaufmann $(\bowtie)$

Clinic for Gynecology, CCM/CBF,

Charité-Universitaetsmedizin Berlin, Gynäkologie mit

Hochschulambulanz, Charité Campus Benjamin Franklin,

Hindenburgdamm 30,

12200 Berlin, Germany

e-mail: andreas.kaufmann@charite.de

\section{A. E. Albers}

Clinic for Otorhinolaryngology, CBF,

Charité-Universitaetsmedizin Berlin,

12200 Berlin, Germany

\section{Introduction}

Human Papillomavirus (HPV) is the most common sexually transmitted agent worldwide and comprises more than 150 genotypes. Persistent infection with high-risk (oncogenic) HPV genotypes, most commonly type 16 and 18 , is responsible for all of the cervical carcinogenesis, and $5.2 \%$ of the total global cancer burden, including cancers of the cervix, penis, vagina, vulva, anus, and oropharynx [1]. Recently, an association between HPV and breast cancer has also been proposed [2]. However, not all persistent infections progress to precancerous (high-grade) lesions, and not all infections with high-risk HPV develop into cancer. Several studies of prevalent HPV infection have concluded that HPV is mainly a transient infection [3-5]. For women who do develop cervical cytology abnormalities, approximately $75 \%$ of low-grade lesions in adults and $90 \%$ of low-grade lesions in adolescents resolve spontaneously [6]. The longer an HPV infection persists, the less probable is a spontaneous clearance of the infection [7].

The most successful strategy for cervical cancer prevention has been the implementation of screening strategies for precancerous cervical lesions (i.e., high-grade dysplasia or cervical intraepithelial neoplasia [CIN] 2/3) utilizing conventional Pap smear tests or, more recently, liquid-based cytology (LBC) tests. Cervical cancer incidence rates in the United States decreased by $75 \%$ and mortality by $74 \%$ in the 50 years following the introduction of cervical cytology in 1949 [8]. However, all screening programs require health infrastructure and financial resources that are not available in most developing countries [9]. In addition, they reduce mortality from cervical neoplasia, but in the first place do not prevent development of precancerous high-grade (CIN 2/3) and persistent low-grade lesions (CIN 1 ), requiring treatment that mainly consists of extirpative or 
ablative procedures aimed at removing visible lesions. These procedures do not target the causative HPV infection and patients with CIN who have undergone ablative surgery or even a hysterectomy may still have HPV infection, which may result in recurrent or persistent CIN [10, 11]. Taken together, HPV-targeted, less invasive treatment methods for precancerous lesions are urgently needed.

HPV infection of the genital tract gives rise to a slow and modest but measurable serum or local antibody response. However, not all individuals infected with HPV develop measurable HPV antibodies, but more than half of all infected subjects will seroconvert [12]. A detectable seroconversion requires sufficient antigenic exposure associated with either a high viral load or persistent infection with HPV [13]. Humoral immunity is directed against conformational epitope(s) derived from the major capsid protein L1 displayed on the outer surface of the intact virus particle and is merely genotype specific [14]. Seropositive individuals are only partially protected against further HPV infection.

Recently available prophylactic vaccines have proven to prevent nearly $100 \%$ of infections and dysplasia induced by the respective vaccine type HPVs. However, they do not target all high-risk HPV types, have a limited crossprotection to other HPV types that cause cancer, have no therapeutic effect, or protect women who are already infected with HPV at the time of vaccination. In addition, their efficacy depends on high vaccination coverage and is not likely to be realized for many years [15].

Therefore, therapeutic HPV vaccines will continue to be an area of intense investigation as a treatment modality for persistent infection and inducing regression of precancerous lesions or remission of advanced cancer.

\section{Challenges and Advancements}

The past two decades have seen a multitude of clinical trials with experimental candidate therapeutic vaccines. Many vaccination strategies were employed and generally proved to be safe and, in most trials, also immunogenic. Clinical responses, however, were sparse or completely lacking. This was not expected initially because cervical cancer has unique HPV-derived antigens that are not tolerized due to thymic negative selection like self-antigens and immune responses had been demonstrated in patients before. It turned out that specific immune evasion and immune resistance mechanisms acquired by the tumor cells during persistent infection and the year-long progression to cancer are responsible for the disappointing clinical results. These mechanisms and how they can be overcome were investigated in detail recently (see further in article), and to some extent, the use of potent adjuvants and the performance of clinical trials in patients with premalignant (not yet immuneescaped) lesions led to more promising clinical results.

In the past 2 years, a more detailed knowledge of the role of the immunosuppressive regulatory $\mathrm{T}$ cells (Tregs) and toll-like receptors (TLRs) in the progression and treatment of HPV-associated diseases has created new strategies for the development of immunotherapy.

Tregs are CD25hi Foxp3+ CD4+ T cells that function to suppress the pathogenic immune responses mediated by effector $\mathrm{T}$ cells and play key roles in controlling immunoreactions [16]. Recently, their interaction with HPV therapeutic vaccines has been further explored. Tregs are found with higher frequency in persistently HPV-infected patients. They play a pivotal role in the suppression of antiviral $\mathrm{T}$ cell responses and severely hamper the efficacy of the therapeutic vaccines $[17,18]$. CD4+ HPV-specific Treg clones have been isolated from HPV-induced intraepithelial lesions, HPV-induced cancers, and their draining lymph nodes [19]. In addition, analyses showed that HPV therapeutic vaccines themselves can induce and/or boost Tregs and that these vaccine-induced Tregs are associated with early treatment failure $[20 \bullet \cdot$. Moreover, it has been shown that repeated vaccinations may increase Treg frequencies [21]. In contrast, selective depletion of Tregs in vivo has already proved effective for tumor rejection in mice and humans. In this respect, our own research showed that combining an adenovirus-based therapeutic vaccination and Treg depletion can lead to the complete and permanent eradication of HPV-expressing tumors in a mouse tumor model [22•]. In patients with condylomata acuminata, Treg depletion using low-dose cyclophosphamide ameliorates the local immune milieu of the lesion site and successfully prevents recurrence of such lesions [23•]. In addition, Liu et al. [24] reported that the combination of adenovirus-mediated expression of immunostimulatory proteins with systemic Treg depletion resulted in a significant delay of tumor growth and even suppressed distant tumor outgrowth in a cervical cancer mouse model. These results showed that therapeutic vaccines in combination with agents capable to target and eliminate Tregs-mediated immunosuppression might be a promising approach to improve HPV-directed immunotherapy. However, other studies in this direction indicated that depletion of Treg in a mouse model of cervical carcinoma did not enhance the immune response induced by the Semliki Forest viral vaccine SFVeE6/7 immunization, suggesting that the SFVeE6/7 vaccine may not require additional immune clearance interventions to modulate Treg activity [25].

A complementary approach is to further enhance vaccine-induced immune responses by potent adjuvants. TLRs are the best-characterized family of patternrecognition receptors that detect microbe-derived substances. When activated, they stimulate the production of 
proinflammatory cytokines and chemokines to increase the host's ability to eliminate pathogens [26]. TLRs are expressed on a wide variety of cells, including B cells, specific types of $\mathrm{T}$ cells, monocytes, dendritic cells (DC), macrophages, certain epithelial cells, and many other innate immune cells [27]. In recent years, the role of TLRs in HPV persistence, clearance, and HPV-associated cancer progression has become an active field of research with many exciting results. Dampened TLR expression in the cervical mucosa is a type-specific mechanism by which HPV16 interferes with innate immune responses. This contributes to viral persistence. On the other hand, TLR upregulation and resultant cytokine induction is important in HPV clearance [28•]. In a similar study, the downregulation of TLR4 was found to be associated with the progression of cervical neoplasia [29]. Moreover, upregulation of TLR in the stroma and not in the epithelium might contribute to the persistence of HPV and ultimately promote the progression of the HPV associated lesions [30••]. Therefore, TLRs and their signaling pathways may act as attractive targets for therapeutics.

For example, agonists that target the TLRs recently have been used as adjuvants to enhance the efficacy of vaccines. Imiquimod was the first approved topically active TLR7 agonist against HPV. It was demonstrated to change the microenvironment of HPV-induced vulvar intraepithelial neoplasia (VIN) and this resulted in viral clearance, normalization of immune cell counts, and a complete regression of the lesion [31]. In a phase 2 study, therapeutic HPV vaccination (TA-CIN, fusion protein HPV16 E6E7L2) in conjunction with imiquimod in patients with VIN grades 2 and 3 led to clinical response in some of the patients with significant systemic HPV-specific immune response and local infiltration of CD4+ and CD8+ T cells but not Tregs in the lesions of these patients [32]. In addition, it was described that some TLR3/4 agonists could promote the maturation of E7-loaded myeloid-derived DCs and their ability to induce $\mathrm{T}$ helper type 1 polarization, augmenting an E7-specific T cell response [33]. These TLR3/4 agonists could significantly enhance antitumor activity and might be a promising adjuvant for HPV therapeutic vaccines against HPV infections and lesions.

\section{Recently Applied Therapeutic Vaccine Strategies}

Initial vaccine strategies used HLA-restricted minimal peptide epitopes, recombinantly expressed proteins, recombinant viral vectors, autologous DCs, and, recently, DNA expression plasmids. Recently investigated strategies are summarized in Table 1. The choice of the target antigen is extremely important for designing therapeutic vaccines. Until now, most therapeutic HPV vaccines have targeted one genotype of HPV, or at most two. The viral oncogenes (HPV E6 and E7) represent ideal antigens for therapeutic HPV vaccination because their sustained expression is required for the maintenance of the cancerous phenotype [34]. However, some studies using highly sensitive and multiplexed HPV tests are beginning to reveal that in many cases of advanced cervical dysplasia [35], and even in cervical cancer [36], there are multiple genotypes of high-risk HPV present in the infected tissue. In such a scenario, targeting only a single HPV genotype would likely be ineffective as a therapeutic approach in some cases. Thus, researchers are seeking to develop novel broad-spectrum vaccines. A novel, broad-spectrum, recombinant fusion protein-based vaccine is directed at the E7 proteins of five of the most prevalent high-risk HPV genotypes worldwide (HPV16, $18,31,45$, and 52). Together they account for more than $80 \%$ of all HPV-associated cancers and elicit strong, multigenotype specific CD8 $\mathrm{T}$ cell immunity when administered to mice in combination with adjuvants comprised of agonists of the TLR3 or TLR9 family of innate immune receptors [37•].

Protein- and long peptide-based vaccines have become attractive approaches in generating antigen-specific immunotherapy because of their safety, simplicity, and coverage of many human leukocyte antigen (HLA) restrictions for presentation [38]. However, the use of protein or long peptides as vaccines has been slowed by the need for strong adjuvants because of their low immunogenicity. Recently, several new protein and peptide-based vaccines against HPV E6 and/or E7 have overcome this obstacle and showed satisfying results in preclinical models. A HPV16E6/E7 long peptide vaccine adjuvanted with Montanide ISA-51 (SEPPIC, Puteaux, France), a detoxified Freunds adjuvant, demonstrated an extraordinary efficacy of $79 \%$ of clinical responses in a clinical trial in patients with persistent VIN lesions. The most important observation is that a measurable antigen-specific $\mathrm{T}$ cell response that clearly correlated with the clinical responses was induced [39••]. Progress also has been demonstrated in preclinical models. A new protein vaccine using autologous HSP70 as HPV16 E7 antigen vector could elicit a potent E7-specific anti-tumor effect without any adjuvantation in the mouse cervical cancer model TC-1 [40]. In another study, a novel fusion protein-based vaccine comprising LALF32-51, a cellpenetrating and immunostimulatory peptide, linked to HPV16 E7 antigen could significantly improve the presentation of E7-derived peptides to T cells in vitro, and consequently induced a potent E7-specific cell-mediated immune response and inhibiting TC-1 tumor growth [41].

Recently, a new HPV fusion peptide composed of Tat4957 (amino acids 49-57) and the cytotoxic $\mathrm{T}$ lymphocyte (CTL) epitope HPV16E7 (amino acids 49-57) combined with GM-CSF expression plasmid application has been 


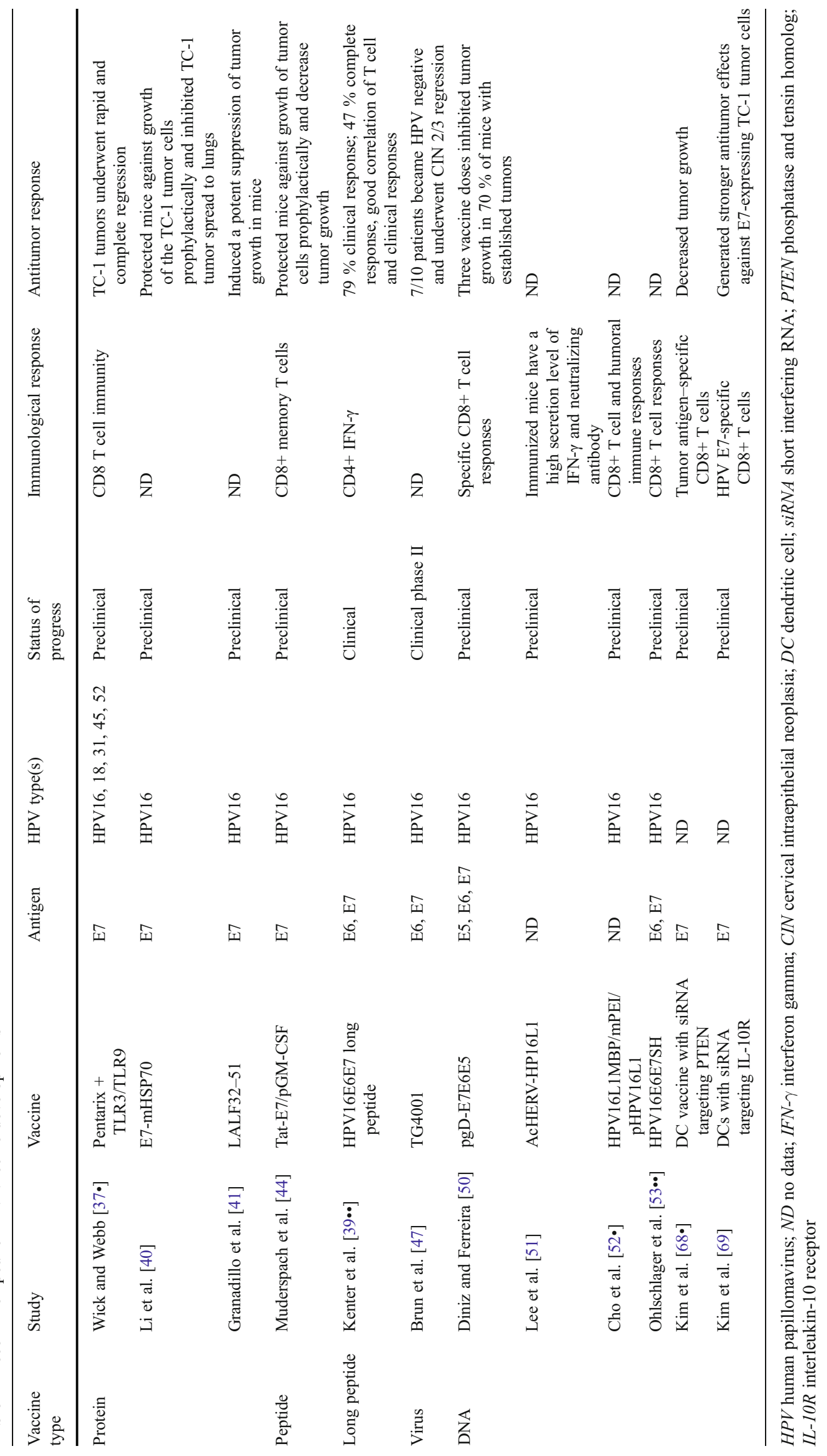


shown to be effective in eliciting potent long-lasting CD8+ memory T cells immune responses ex vivo and in vivo [42]. In patients suffering from HPV-associated lesions, peptidebased vaccines have been tested for their therapeutic efficacy but showed only moderate immune and clinical responses $[43,44]$. It will be interesting to see if the new approaches will be more effective.

Because of their high infection efficiency and excellent expression of antigens in the infected cells, recombinant viral vectors are considered an alluring choice for the delivery of HPV protein [45]. The disadvantage of some viral vectors is the development of host-induced neutralizing antibodies to the vector itself, thus limiting its continued use. The nature of viral vaccine platforms renders them exceptionally suitable for multicenter randomized trials [46]. In a recent phase 2 clinical trial, patients with HPV16-related CIN 2/3 were treated three times at weekly intervals with TG4001 vaccine, consisting of an attenuated recombinant vaccinia virus containing the sequence for modified HPV16 E6 and E7, and the human interleukin (IL)-2 gene. At month 6 , regression of the CIN 2/3 lesion and the clearance of HPV 16 infection were seen in 7 of 10 patients [47]. Future directions will involve its efficacy in further phase 3 clinical trials of HPV-associated lesions and whether its antitumor response will be enhanced in combination with neoadjuvant chemotherapy or other adjunct forms of therapy.

DNA vaccines have the advantage of being safe and easy to produce, and can be administered repeatedly to the same individual without loss of efficacy [48]. However, naked DNA suffers from insufficient intrinsic specificity for antigen-presenting cells (APCs) and has a limited ability to amplify and spread in vivo. Therefore, various strategies to enhance therapeutic HPV DNA vaccines are currently under active investigation, including alternative immunization routes and delivery methods. The gene gun is a ballistic device that enables delivered DNA to directly transfect keratinocytes and epidermal Langerhans cells. These events stimulate DC maturation and migration to the local lymphoid tissue, where they prime $\mathrm{T}$ cells for antigen-specific immune responses [48]. The delivery of HPV DNA vaccines by gene gun was shown to be the most dose-efficient method of vaccine administration compared to needle intramuscular (i.m.) and Biojector (Bioject Medical Technologies, Inc., Tualatin, OR) administrations [49]. In a recent study, a newly developed DNA vaccine encoding herpes simplex virus type 1 (HSV-1) glycoprotein D genetically fused to three HPV16 oncoproteins (E5, E6, and E7), by intradermal (i.d.) administration using a gene gun, induced strong antigen-specific $\mathrm{CD} 8+\mathrm{T}$ cell responses in vaccinated mice and conferred enhanced antitumor protection with much lower DNA loads when compared to the i.m. delivered DNA vaccine. Significantly stronger therapeutic antitumor effects were observed after i.d. immunization with a single dose of pgD-E7E6E5 co-administration of DNA vectors encoding a co-stimulatory signal, such as granulocyte-macrophage colony-stimulating factor (GMCSF) or IL-12 [50].

A therapeutic vaccine in widespread use should be acceptably cost effective and highly effective in clearing existing HPV infection. Several novel delivery systems appeared to have higher cellular immunogenicity and much less cost compared to the widely studied methods of introducing foreign genes into mammalian cells such as chemicalbased procedures, electroporation, gene-gun, and mammalian viral vector-based systems [48]. Recently, a recombinant baculovirus bearing human endogenous retrovirus (HERV) envelope protein, was used as a nano-carrier for HPV-16L1 DNA vaccine (AcHERV-HP16L1). Compared with the virus-like particle-based vaccine Gardasil ${ }^{\circledR}$ (Merck \& Co., Whitehouse Station, NJ), mice immunized with AcHERVHP16L1 showed similar high levels of humoral immunity in $\mathrm{IgG} / \mathrm{IgA}$ and in neutralization of HPV pseudovirions. Interestingly, a 450- and 490-fold increase in the interferon (IFN) $-\gamma$ T cell response was observed at 5 and 20 weeks after the first injection, respectively. The recombinant baculovirus used for HPV16L1 gene delivery can induce both humoral and cellular immunity. Therefore, it could potentially be developed as an efficient prophylactic and therapeutic vaccine [51]. In another report, HPV16L1MBP/mPEI/pHPV16L1, simultaneously carrying a protein vaccine along with its corresponding DNA vaccine, could trigger the highest levels of humoral immune responses among various vaccination groups and enhanced the number of interferon-producing CD8+ $\mathrm{T}$ cells in a mouse model [52•].

Our own approach was to combine the antigencarrying plasmid (HPV16E7SH) with expression plasmids for cytokines (IL-2, IL-12, GM-CSF, IFN- $\gamma$, macrophage inflammatory protein (MIP) $1-\alpha$ ) as adjuvants. Given in different combinations and time points around the vaccination gene-encoded MIP- $1 \alpha$ applied 5 days before E7SH-immunization combined with IFN- $\gamma$ or IL-12 (3 days) or IL-2 (5 days) postimmunization led to a significantly enhanced tumor response that was clearly associated with granzyme B secretion and target cells lysis $[53 \bullet \bullet$.

DC-based vaccines are potent options for cancer immunotherapy because DCs are the most important professional APCs capable of priming naïve $\mathrm{T}$ cells and can serve as natural adjuvants [54,55]. A number of strategies have been developed including the use of different vectors, pulsing of DC with proteins and peptides, or transfecting DC with DNA or RNA to enhance the potency of therapeutic 
vaccines for HPV [56-61]. However, the immune responses elicited by the first-generation DC vaccines might not be of the quality required to allow the rejection of bulky tumors because of some specific problems such as a short lifespan of DC, few DCs from the vaccination site migrating to the lymph node, the strongly immunosuppressive cytokine microenvironment within the tumor, or the recruitment of immunosuppressive cells like regulatory $\mathrm{T}$ cells [62-67]. Several strategies have been used to improve DC-based vaccines. Kim et al. [68・] reported that a DC vaccine combined with a small interfering RNA (siRNA) targeting phosphatase and tensin homologue (PTEN) led to an increase of in vitro $\mathrm{T}$ cell activation activity and in vivo migration to a draining lymph node, respectively. Moreover, these PTEN siRNA-transfected DCs (DC/siPTEN) acquired an increased survival and protection from apoptotic death caused by GMCSF deprivation or antigen-specific CD8 $+\mathrm{T}$ cell killing. Most importantly, DC/siPTEN generated more tumor antigen-specific $\mathrm{CD} 8+\mathrm{T}$ cells and stronger antitumor effects in vaccinated mice than did control DCs (DC/ siGFP). In another report, DCs modified with siRNA targeting against IL-10R generated more HPV E7specific CD8 $+\mathrm{T}$ cells and stronger antitumor effects against E7-expressing TC-1 tumour cells in vaccinated mice than DC/siGFP, as well as DC/silL-10 [69]. These results demonstrated that using siRNA for interference with immunosuppressive cytokines could enhance DCbased vaccine potency. We may infer that the DC vaccines, combined with other therapies that target many different immunosuppressive agents and signaling pathways, may lead to even better antitumor efficacy.

\section{Combinational Approaches to Enhance HPV Therapeutic Vaccine Potency}

Each strategy for delivery of therapeutic HPV vaccines has its own advantages and potential disadvantages. Optimally designed cancer vaccines should combine the best tumor antigens with the most effective immunotherapy agents, delivery strategies, and administration routes to achieve positive clinical results. Ideally, this is first investigated in an uncompromised healthy immune system after safety has been proven and then transferred to patients $[70,71]$.

Adjuvants are incorporated into a vaccine to increase vaccine immunogenicity by potentiating antigen-specific $\mathrm{CD} 8+$ and/or CD4+ $\mathrm{T}$ cell responses, or to increase vaccine effectiveness by impairing one or more immunosuppressive mechanisms that operate at the level of the tumor microenvironment [72-76]. Therefore, recent studies were dedicated to seeking better adjuvants to improve the efficacy of therapeutic HPV vaccines. SA-
4-1BBL, a multifunctional immunomodulator, was used in combination with a HPV16 E7 recombinant proteinbased therapeutic vaccine, and induced complete eradication of established tumors in approximately $70 \%$ of mice [77]. In two other preclinical studies, the combination of 5,6-dimethylxanthenone-4-acetic acid (DMXAA), a vascular disrupting agent [78], with therapeutic HPV16 E7 peptide-based vaccination [79•] or E7 DNA vaccination [80] were demonstrated to enhance the antitumor effect and generate strong E7-specific CD8+ T-cell immune responses. Using the HPV16E7expressing TC-1 tumor model, mice were vaccinated with HPV16 E7 peptide, together with a pan HLA-DR epitope (PADRE) peptide, which is capable of binding to different major histocompatibility complex (MHC) class II molecules with high-affinity [81] and the TLR3 ligand poly(I:C) adjuvant, a strong inducer of type I IFN [82]. PADRE peptide and poly (I:C) were used to enhance the activation of $\mathrm{CD} 4+\mathrm{T}$ helper cells and $\mathrm{DC}$, respectively, which altogether generated better E7-specific CD8+T cell immune responses as well as antitumor effects against TC-1 tumors [83]. In another study, an adenovirus-based $\mathrm{T}$ cell vaccine (Ad-p14) in combination with treatment by antiglucocorticoidinduced tumor necrosis factor receptor (GITR) antibody resulted in complete and permanent eradication of $100 \%$ of TC-1 tumors in mice [22•]. Moreover, a recent study has used the natural killer $\mathrm{T}$ cell (NKT) ligand $\alpha$-galactosylceramide ( $\alpha$-GalCer) as an adjuvant with DNA vaccination against HPV16 oncoprotein E7 to generate high numbers of E7-specific CD8+ T cells protective against the transplantable TC-1 (HPV-16+) tumor model [84•]. These new adjuvants trigger innate and adaptive immunity by targeting different innate receptors or pathways.

Electroporation represents one way of improving DNA penetration into cells and enhances the magnitude of gene expression. Two recent studies have demonstrated that combining adjuvant $\mathrm{CpG}$ motifs and electroporation can trigger an enhanced cellular immune responses and stronger regression of tumors [85, 86•, 87••]. Therefore, selecting an appropriate adjuvant and delivery route may maximize the antitumor potency of therapeutic vaccines.

Until now, the delivery of HPV therapeutic vaccines using i.m., subcutaneous, and i.d. administration has been shown to be the most efficient routes of vaccine administration.

However, bone marrow (BM) was recently shown to be a unique microenvironment that supported robust proliferation and recalled responses of memory $\mathrm{T}$ cells, particularly within the $\mathrm{CD} 8+\mathrm{T}$ cell subset. The population of $\mathrm{CD} 8+$ effector memory $\mathrm{T}$ cells within the human BM has a hybrid phenotype and enhanced cytotoxic 
function (reviewed in [86•]) [88]. Therefore, BMderived $\mathrm{T}$ cells may be the ideal effectors for HPV therapeutic vaccine strategies. Fresnay et al. [89•] reported that direct intra-BM (IBM) vaccination with a class I HPV16 E7 epitope induced large numbers of activated, IFN- $\gamma$-producing E7-specific lymphocytes in mice. In the therapeutic setting, IBM vaccination induced tumor regression in 3 of 10 vaccinated mice and delayed tumor growth in the remaining animals significantly.

Mucosal immunization may be important for protection against pathogens whose transmission and pathogenesis target mucosal tissue [90]. Sexually-transmitted HPV types are prototypic mucosal pathogens that infiltrate epithelial lesions and infect cells of mucosal or squamous epithelia [91]. Therapeutic responses against HPV infection and cancer might be induced systemically and/or at mucosal surfaces by activating the mucosal immune system, which has been established as an ideal target site for some vaccines [92]. Recently, C57BL/6 mice were orally exposed to recombinant Lactobacillus casei expressing HPV16 E7 (LacE7). The induction of specific mucosal E7 type $1 \mathrm{~T}$ cell with responses was greater after oral immunization with LacE7 when compared to subcutaneous or i.m. antigen delivery. Oral immunization with Lactobacillus-based vaccines was also able to induce mucosal cytotoxic cellular immune responses [93]. In a similar study, in the TC-1 tumor model, animals receiving the orally administered $L$. casei-PgsA-E6 vaccine generated systemic and local cellular immune responses and showed reduced tumor size and increased survival rates [94]. Thus, new administration routes may potentially enhance therapeutic effects of individual HPV therapeutic vaccines.

\section{Conclusions}

In the past few years, a significant progress in therapeutic vaccines has been made in experimental preclinical research and initial clinical trials. However, now it is important to pave the way for further clinical trials to see these vaccines' true efficacy against HPV-associated lesions, especially against advanced cancer. Advances in understanding the mechanisms of the cellular and molecular nature of innate and adaptive immunity have provided insights into how adjuvants might be improved to enhance immunity and achieve an adequate response. The current wealth of preclinical and clinical information predicts that an ideal HPV therapeutic vaccine should activate both innate and adaptive immunity. Therefore, this would prevent persistent HPV infection from progressing toward advanced cancer or even promote clearance of HPV infection.
Disclosures No potential conflicts of interest relevant to this article were reported.

\section{References}

Papers of published papers of interest have been highlighted as:

- Of importance

•• Of major importance

1. Parkin DM. The global health burden of infection-associated cancers in the year 2002. Int J Cancer. 2006;118:3030-44.

2. Heng B, Glenn WK, Ye Y, et al. Human papilloma virus is associated with breast cancer. Brit J Cancer. 2009;101:1345-50.

3. Romney SL, Ho GYF, Palan PR, et al. Effects of beta-carotene and other factors on outcome of cervical dysplasia and human papillomavirus infection. Gynecol Oncol. 1997;65:483-92.

4. Hildesheim A, Schiffman MH, Gravitt PE, et al. Persistence of type-specific human papillomavirus infection among cytologically normal women. J Infect Dis. 1994;169:235-40.

5. Devilliers EM, Wagner D, Schneider A, et al. Human papillomavirus DNA in women without and with cytological abnormalities - results of a 5-year follow-up-study. Gynecol Oncol. 1992;44:33-9.

6. Cuzick J, Castanon A, Sasieni P. Predicted impact of vaccination against human papillomavirus $16 / 18$ on cancer incidence and cervical abnormalities in women aged 20-29 in the UK. Br J Cancer. 2010;102:933-9.

7. Ho GY, Bierman R, Beardsley L, Chang CJ, Burk RD. Natural history of cervicovaginal papillomavirus infection in young women. N Engl J Med. 1998;338:423-8.

8. Cronje HS. Screening for cervical cancer in developing countries. Int J Gynaecol Obstet. 2004;84(2):101-8.

9. Cronje HS. Screening for cervical cancer in developing countries. Int J Gynaecol Obstet: The Official Organ of the International Federation of Gynaecology and Obstetrics. 2004;84:101-8.

10. Einstein MH, Kadish AS, Burk RD, et al. Heat shock fusion protein-based immunotherapy for treatment of cervical intraepithelial neoplasia III. Gynecol Oncol. 2007;106:453-60.

11. Van Doorslaer K, Reimers LL, Studentsov YY, Einstein MH, Burk RD. Serological response to an HPV16 E7 based therapeutic vaccine in women with high-grade cervical dysplasia. Gynecol Oncol. 2010;116:208-12.

12. Carter JJ, Koutsky LA, Hughes JP, et al. Comparison of human papillomavirus types 16,18 , and 6 capsid antibody responses following incident infection. J Infect Dis. 2000;181:1911-9.

13. Ho GY, Studentsov YY, Bierman R, Burk RD. Natural history of human papillomavirus type 16 virus-like particle antibodies in young women. Cancer Epidemiol Biomark Prev. 2004;13:110-6. A publication of the American Association for Cancer Research, cosponsored by the American Society of Preventive Oncology.

14. Gissmann L. Immunologic responses to human papillomavirus infection. Obstet Gynecol Clin N Am. 1996;23:625.

15. Franco EL, Duarte-Franco E, Ferenczy A. Cervical cancer: epidemiology, prevention and the role of human papillomavirus infection. Can Med Assoc J. 2001;164:1017-25.

16. Mills KHG. Regulatory T cells: friend or foe in immunity to infection? Nat Rev Immunol. 2004;4:841-55.

17. Sakaguchi S. Naturally arising Foxp3-expressing CD25(+) CD4 $(+)$ regulatory T cells in immunological tolerance to self and nonself. Nat Immunol. 2005;6:345-52.

18. Molling JW, de Gruij1 TD, Glim J, et al. CD4(+)CD25(hi) regulatory T-cell frequency correlates with persistence of human 
papillomavirus type 16 and T helper cell responses in patients with cervical intraepithelial neoplasia. Int J Cancer. 2007;121:1749-55.

19. van der Burg SH, Arens R, Melief CJM. Immunotherapy for persistent viral infections and associated disease. Trends Immunol. 2011;32:97-103.

20. • Welters MJ, Kenter GG, de Vos van Steenwijk PJ, et al. Success or failure of vaccination for HPV16-positive vulvar lesions correlates with kinetics and phenotype of induced T-cell responses. Proc Natl Acad Sci U S A. 2010;107:11895-9. This study demonstrates that larger lesions display weaker effector T cell responses and stronger $H P V$-specific regulatory $T$ cell immune responses in patients with HPV-induced vulvar intraepithelial neoplasia grade 3. This finding illustrates that the current strategy of immunotherapy for patients with premalignant lesions may need to be supplemented by means controlling regulatory $T$ cell responses.

21. Schaefer JT, Patterson JW, Deacon DH, et al. Dynamic changes in cellular infiltrates with repeated cutaneous vaccination: a histologic and immunophenotypic analysis. J Transl Med. 2010;8:79.

22. - Hoffmann C, Stanke J, Kaufmann AM, Loddenkemper C, Schneider A, Cichon G. Combining T-cell vaccination and application of agonistic anti-GITR mAb (DTA-1) induces complete eradication of HPV oncogene expressing tumors in mice. J Immunother. 2010;33:136-45. In comparison to all other combinations with adjuvants, the treatment to delete Treg was the only one to induce a complete and lasting therapeutic effect. No tumor regrowth was observed while after other combinations generally tumors recurred.

23. - Cao Y, Zhao J, Yang Z, et al. CD4+FOXP3+ regulatory T cell depletion by low-dose cyclophosphamide prevents recurrence in patients with large condylomata acuminata after laser therapy. Clin Immunol. 2010;136:21-9. This clinical study demonstrated that in patients Tregs can be depleted by cyclophosphamide treatment. This depletion leads to a reduced rate of recurrence, pointing toward a more effective natural immune response after therapy.

24. Liu Y, Tuve S, Persson J, et al. Adenovirus-mediated intratumoral expression of immunostimulatory proteins in combination with systemic Treg inactivation induces tumor-destructive immune responses in mouse models. Cancer Gene Ther. 2011;18:407-18.

25. Walczak M, Regts J, van Oosterhout AJ, et al. Role of regulatory T-cells in immunization strategies involving a recombinant alphavirus vector system. Antivir Ther. 2011;16:207-18.

26. Takeuchi O, Akira S. Pattern recognition receptors and inflammation. Cell. 2010;140:805-20.

27. Liotta F, Angeli R, Cosmi L, et al. Toll-like receptors 3 and 4 are expressed by human bone marrow-derived mesenchymal stem cells and can inhibit their T-cell modulatory activity by impairing notch signaling. Stem Cells. 2008;26:279-89.

28. • Daud II, Scott ME, Ma YF, Shiboski S, Farhat S, Moscicki AB. Association between toll-like receptor expression and human papillomavirus type 16 persistence. Int J Cancer. 2011;128:879-86. This study shows a correlation of reduced TLR expression in persistently HPV infected patients. This may be one reason for an ineffective immune response in patients.

29. Yu L, Wang LT, Li MZ, Zhong JM, Wang Z, Chen SW. Expression of toll-like receptor 4 is down-regulated during progression of cervical neoplasia. Cancer Immunol Immun. 2010;59:1021-8.

30. •• DeCarlo CA, Rosa B, Jackson R, Niccoli S, Escott NG, Zehbe I. Toll-like receptor transcriptome in the HPV-positive cervical cancer microenvironment. Clin Dev Immunol. 2012;2012:785825. This study shows that stromal upregulation of TLRs 3 and 1 may play a role in cervical disease progression. TLRs 3 and 1 may be ideal diagnostic and prognostic markers for the risk of patients with premalignant disease to develop cervical cancer.

31. Terlou A, van Seters M, Kleinjan A, et al. Imiquimod-induced clearance of HPV is associated with normalization of immune cell counts in usual type vulvar intraepithelial neoplasia. Int J Cancer. 2010;127:2831-40.
32. Daayana S, Elkord E, Winters U, et al. Phase II trial of imiquimod and HPV therapeutic vaccination in patients with vulval intraepithelial neoplasia. Brit J Cancer. 2010;102:1129-36.

33. Chen XZ, Mao XH, Zhu KJ, et al. Toll like receptor agonists augment HPV 11 E7-specific $\mathrm{T}$ cell responses by modulating monocyte-derived dendritic cells. Arch Dermatol Res. 2010;302:57-65.

34. zur Hausen H. Papillomaviruses and cancer: from basic studies to clinical application. Nat Rev Cancer. 2002;2:342-50.

35. Moodley M. Update on pathophysiologic mechanisms of human papillomavirus. Curr Opin Obstet Gyn. 2005;17:61-4.

36. Schmitt M, Dondog B, Waterboer T, Pawlita M, Tommasino M, Gheit T. Abundance of multiple high-risk human papillomavirus (HPV) infections found in cervical cells analyzed by use of an ultrasensitive HPV genotyping assay. J Clin Microbiol. 2010;48:143-9.

37. - Wick DA, Webb JR. A novel, broad spectrum therapeutic HPV vaccine targeting the E7 proteins of HPV16, 18, 31, 45 and 52 that elicits potent E7-specific CD8T cell immunity and regression of large, established, E7-expressing TC-1 tumors. Vaccine. 2011;29:7857-66. This paper reports a novel therapeutic vaccine targeting simultaneously five high-risk HPV genotypes. It is capable of eliciting an effective and broad spectrum anti-HPV CD $\mathrm{T}$ cell immunity.

38. Mahdavi A, Monk BJ. Vaccines against human papillomavirus and cervical cancer: promises and challenges. Oncologist. 2005; $10: 528-38$

39. •• Kenter GG, Welters MJ, Valentijn AR, et al. Vaccination against HPV-16 oncoproteins for vulvar intraepithelial neoplasia. N Engl J Med. 2009;361:1838-47. This study showed very clearly for the first time a correlation of an induced T cellular immune response with the positive clinical outcome of the therapeutic vaccine.

40. Li YL, Liu J, Liu JN, Zhang J. Immunization of protein HPV16 E7 in fusion with mouse HSP70 inhibits the growth of TC-1 cells in tumor bearing mice. Vaccine. 2011;29:5959-62.

41. Granadillo M, Vallespi MG, Batte A, et al. A novel fusion proteinbased vaccine comprising a cell penetrating and immunostimulatory peptide linked to human papillomavirus (HPV) type 16 E7 antigen generates potent immunologic and anti-tumor responses in mice. Vaccine. 2011;29:920-30.

42. Tang J, Yin R, Tian Y, et al. A novel self-assembled nanoparticle vaccine with HIV-1 Tat/HPV16 E7 fusion peptide and GM-CSF DNA elicits potent and prolonged CD8 T cell-dependent antitumor immunity in mice. Vaccine. 2012;30:1071-82.

43. Vambutas A, DeVoti J, Nouri M, et al. Therapeutic vaccination with papillomavirus E6 and E7 long peptides results in the control of both established virus-induced lesions and latently infected sites in a pre-clinical cottontail rabbit papillomavirus model. Vaccine. 2005;23:5271-80.

44. Muderspach L, Wilczynski S, Roman L, et al. A phase I trial of a human papillomavirus (HPV) peptide vaccine for women with high-grade cervical and vulvar intraepithelial neoplasia who are HPV 16 positive. Clin Cancer Res. 2000;6:3406-16.

45. $\mathrm{Su} \mathrm{JH}, \mathrm{Wu} \mathrm{A}$, Scotney E, et al. Immunotherapy for cervical cancer: research status and clinical potential. BioDrugs. 2010;24:109-29.

46. Larocca C, Schlom J. Viral vector-based therapeutic cancer vaccines. Cancer J. 2011;17:359-71.

47. Brun JL, Dalstein V, Leveque J, et al. Regression of high-grade cervical intraepithelial neoplasia with TG4001 targeted immunotherapy. Am J Obstet Gynecol. 2011;204:169 e1-8.

48. Lin K, Roosinovich E, Ma B, Hung CF, Wu TC. Therapeutic HPV DNA vaccines. Immunol Res. 2010;47:86-112.

49. Trimble C, Lin CT, Hung CF, et al. Comparison of the CD8+ T cell responses and antitumor effects generated by DNA vaccine administered through gene gun, biojector, and syringe. Vaccine. $2003 ; 21: 4036-42$. 
50. Diniz MO, Ferreira LCS. Enhanced anti-tumor effect of a gene gun-delivered DNA vaccine encoding the human papillomavirus type 16 oncoproteins genetically fused to the herpes simplex virus glycoprotein D. Braz J Med Biol Res. 2011;44:421-7.

51. Lee HJ, Park N, Cho HJ, et al. Development of a novel viral DNA vaccine against human papillomavirus: AcHERV-HP16L1. Vaccine. 2010;28:1613-9.

52. - Cho HJ, Han SE, Im S, et al. Maltosylated polyethyleniminebased triple nanocomplexes of human papillomavirus $16 \mathrm{~L} 1$ protein and DNA as a vaccine co-delivery system. Biomaterials. 2011;32:4621-9. Nanoparticles facilitate the uptake of antigen and DNA vaccine that is critical for the strength of the induced immune response.

53. •- Ohlschlager P, Quetting M, Alvarez G, Durst M, Gissmann L, Kaufmann AM. Enhancement of immunogenicity of a therapeutic cervical cancer DNA-based vaccine by co-application of sequenceoptimized genetic adjuvants. Int J Cancer. 2009;125:189-98. The combination and consecutive application of co-administered expression plasmids for cytokines and a DNA vaccine to HPV16 showed enhanced efficacy.

54. Bonifaz LC, Bonnyay DP, Charalambous A, et al. In vivo targeting of antigens to maturing dendritic cells via the DEC-205 receptor improves T cell vaccination. J Exp Med. 2004;199:815-24.

55. Santin AD, Bellone S, Roman JJ, Burnett A, Cannon MJ, Pecorelli $\mathrm{S}$. Therapeutic vaccines for cervical cancer: dendritic cell-based immunotherapy. Curr Pharm Des. 2005;11:3485-500.

56. Tsen SWD, Paik AH, Hung CF, Wu TC. Enhancing DNA vaccine potency by modifying the properties of antigen-presenting cells. Expert Rev Vaccines. 2007;6:227-39.

57. Kim JH, Kang TH, Noh KH, et al. Enhancement of dendritic cellbased vaccine potency by anti-apoptotic siRNAs targeting key proapoptotic proteins in cytotoxic CD8(+) T cell-mediated cell death. Immunol Lett. 2009;122:58-67.

58. Benencia F, Courreges MC, Coukos G. Whole tumor antigen vaccination using dendritic cells: comparison of RNA electroporation and pulsing with UV-irradiated tumor cells. J Transl Med. 2008;6.

59. Santin AD, Bellone S, Gokden M, Cannon MJ, Parham GP. Vaccination with HPV-18 E7-pulsed dendritic cells in a patient with metastatic cervical cancer. New Engl J Med. 2002;346:1752-3.

60. Santin AD, Bellone S, Palmieri M, et al. HPV16/18 E7-pulsed dendritic cell vaccination in cervical cancer patients with recurrent disease refractory to standard treatment modalities. Gynecol Oncol. 2006;100:469-78.

61. Santin AD, Bellone S, Palmieri M, et al. Human papillomavirus type 16 and 18 E7-pulsed dendritic cell vaccination of stage IB or IIA cervical cancer patients: a phase I escalating-dose trial. J Virol. 2008;82:1968-79.

62. Bergmann C, Strauss L, Wieckowski E, et al. Tumor-derived microvesicles in sera of patients with head and neck cancer and their role in tumor progression. Head Neck-J Sci Spec. 2009;31:371-80.

63. Albers AE, Ferris RL, Kim GG, Chikamatsu K, DeLeo AB, Whiteside TL. Immune responses to p53 in patients with cancer: enrichment in tetramer+p53 peptide-specific $\mathrm{T}$ cells and regulatory $\mathrm{T}$ cells at tumor sites. Cancer Immunol Immun. 2005;54:1072-81.

64. Schaefer C, Kim GG, Albers A, Hoermann K, Myers EN, Whiteside TL. Characteristics of CD4+CD25+ regulatory T cells in the peripheral circulation of patients with head and neck cancer. Brit $\mathrm{J}$ Cancer. 2005;92:913-20.

65. de Vries IJM, Krooshoop DJEB, Scharenborg NM, et al. Effective migration of antigen-pulsed dendritic cells to lymph nodes in melanoma patients is determined by their maturation state. Cancer Res. 2003;63:12-7.

66. Palucka K, Ueno H, Banchereau J. Recent developments in cancer vaccines. J Immunol. 2011;186:1325-31.
67. Zou WP. Immunosuppressive networks in the tumour environment and their therapeutic relevance. Nat Rev Cancer. 2005;5:263-74.

68. • Kim JH, Kang TH, Noh KH, et al. Enhancement of DC vaccine potency by activating the PI3K/AKT pathway with a small interfering RNA targeting PTEN. Immunol Lett. 2010;134:47-54. This paper demonstrates it is possible to modulate signalling pathways by siRNA and thereby enhancing T cell activation.

69. Kim JH, Kang TH, Noh KH, et al. Blocking the immunosuppressive axis with small interfering RNA targeting interleukin (IL)-10 receptor enhances dendritic cell-based vaccine potency. Clin Exp Immunol. 2011;165:180-9.

70. de Jong A, O’Neill T, Khan AY, et al. Enhancement of human papillomavirus (HPV) type 16 E6 and E7-specific T-cell immunity in healthy volunteers through vaccination with TA-CIN, an HPV16 L2E7E6 fusion protein vaccine. Vaccine. 2002;20:3456-64.

71. Hallez S, Simon P, Maudoux F, et al. Phase I/II trial of immunogenicity of a human papillomavirus (HPV) type 16 E7 protein-based vaccine in women with oncogenic HPV-positive cervical intraepithelial neoplasia. Cancer Immunol Immun. 2004;53:642-50.

72. Fioretti D, Iurescia S, Fazio VM, Rinaldi M. DNA vaccines: developing new strategies against cancer. J Biomed Biotechnol. 2010.

73. Schiller M, Metze D, Luger TA, Grabbe S, Gunzer M. Immune response modifiers-mode of action. Exp Dermatol. 2006;15:331-41.

74. Schon MP, Schon M. Immune modulation and apoptosis induction: two sides of the antitumoral activity of imiquimod. Apoptosis: An International Journal on Programmed Cell Death. 2004;9:291-8.

75. Christensen D, Korsholm KS, Andersen P, Agger EM. Cationic liposomes as vaccine adjuvants. Expert Rev Vaccines. 2011;10:513-21.

76. Huang SJ, Hijnen D, Murphy GF, et al. Imiquimod enhances IFNgamma production and effector function of $\mathrm{T}$ cells infiltrating human squamous cell carcinomas of the skin. J Invest Dermatol. 2009;129:2676-85.

77. Sharma RK, Srivastava AK, Yolcu ES, et al. SA-4-1BBL as the immunomodulatory component of a HPV-16 E7 protein based vaccine shows robust therapeutic efficacy in a mouse cervical cancer model. Vaccine. 2010;28:5794-802.

78. Cai SX. Small molecule vascular disrupting agents: potential new drugs for cancer treatment. Recent Pat Anti-Canc. 2007;2:79-101.

79. - Zeng Q, Peng SW, Monie A, et al. Control of cervicovaginal HPV-16 E7-expressing tumors by the combination of therapeutic HPV vaccination and vascular disrupting agents. Hum Gene Ther. 2011;22:809-19. This paper demonstrates that the combination of 5,6-dimethylxanthenone-4-acetic acid (DMXAA) and peptide vaccination enhances immune responses as well as antitumor effects. This paper shows that a vascular disrupting agent may be an important adjuvant for HPV therapeutic vaccine.

80. Peng SW, Monie A, Pang XW, Hung CF, Wu TC. Vascular disrupting agent DMXAA enhances the antitumor effects generated by therapeutic HPV DNA vaccines. J Biomed Sci. 2011;18.

81. Alexander J, Sidney J, Southwood S, et al. Development of high potency universal DR-restricted helper epitopes by modification of high-affinity DR-blocking peptides. Immunity. 1994;1:751-61.

82. Kato H, Takeuchi O, Sato S, et al. Differential roles of MDA5 and RIG-I helicases in the recognition of RNA viruses. Nature. 2006;441:101-5.

83. Wu CY, Monie A, Pang XW, Hung CF, Wu TC. Improving therapeutic HPV peptide-based vaccine potency by enhancing CD4+ T help and dendritic cell activation. J Biomed Sci. 2010;17.

84. - Kim D, Hung CF, Wu TC, Park YM. DNA vaccine with alphagalactosylceramide at prime phase enhances anti-tumor immunity after boosting with antigen-expressing dendritic cells. Vaccine. 2010;28:7297-305. This article details that a prime boost strategy with different therapeutic vaccines enhances immunogenicity of the initial DNA vaccine priming. 
85. McNaughton BR, Cronican JJ, Thompson DB, Liu DR. Mammalian cell penetration, siRNA transfection, and DNA transfection by supercharged proteins. Proc Natl Acad Sci USA. 2009;106:6111-6.

86. - Kang TH, Monie A, Wu LSF, Pang XW, Hung CF, Wu TC. Enhancement of protein vaccine potency by in vivo electroporation mediated intramuscular injection. Vaccine. 2011;29:1082-9. This article discusses enhancement of protein vaccine uptake by an electroporation device that can be used in living organisms. This may become a strategy to load dendritic cells with antigens in situ.

87. •• Ohlschlager P, Spies E, Alvarez G, Quetting M, Groettrup M. The combination of TLR-9 adjuvantation and electroporationmediated delivery enhances in vivo antitumor responses after vaccination with HPV-16 E7 encoding DNA. Int J Cancer. 2011;128:473-81. The uptake of a DNA vaccine into antigen presenting cells and their activation by a TLR agonist is enhanced by an in vivo electroporation. This paper proves that in vivo DNA transfection leads to stronger immune responses.

88. Wood AHD, Zhang XY, Farber DL, Strome SE. CD8(+) memory $\mathrm{T}$ lymphocytes from bone marrow immune function and therapeutic potential. Crit Rev Immunol. 2007;27:527-37.

89. - Fresnay S, Zhang XY, Strome SE, Sewell DA. Bone marrow vaccination: a novel approach to enhance antigen specific antitumor immunity. Vaccine. 2011;29:8599-605. Direct intrabone marrow vaccination induces a high percentage of E7specific memory $T$ cell populations in the bone marrow, which protects mice against tumor development. This study demonstrates bone marrow may be an ideal target for cancer immunotherapy strategies.

90. Graham BS, Kines RC, Corbett KS, et al. Mucosal delivery of human papillomavirus pseudovirus-encapsidated plasmids improves the potency of DNA vaccination. Mucosal Immunol. 2010;3:475-86.

91. Lowy DR, Schiller JT. Prophylactic human papillomavirus vaccines. J Clin Invest. 2006;116:1167-73.

92. Bolhassani A, Safaiyan S, Rafati S. Improvement of different vaccine delivery systems for cancer therapy. Molec Cancer. 2011;10:3.

93. Adachi K, Kawana K, Yokoyama T, et al. Oral immunization with a Lactobacillus casei vaccine expressing human papillomavirus (HPV) type $16 \mathrm{E} 7$ is an effective strategy to induce mucosal cytotoxic lymphocytes against HPV16 E7. Vaccine. 2010;28:2810-7.

94. Lee TY, Kim YH, Lee KS, et al. Human papillomavirus type 16 E6-specific antitumor immunity is induced by oral administration of HPV16 E6-expressing Lactobacillus casei in C57BL/6 mice. Cancer Immunol Immun. 2010;59:1727-37. 